\title{
Projective splitting obstruction groups for one-sided submanifolds
}

\author{
Yu. V. Muranov and I. Hambleton
}

\begin{abstract}
A geometric diagram of groups, which consists of groups equipped with geometric antistructures, is a natural generalization of the square of fundamental groups arising in the splitting problem for a one-sided submanifold. In the present paper the groups $L S_{*}$ and $L P_{*}$ of such diagrams are defined and the properties of these groups are described. Methods for the computation of $L S_{*}^{p}, L P_{*}^{p}$-groups and natural maps in diagrams of exact sequences are developed in the case of a geometric diagram of finite 2-groups.

Bibliography: 21 titles.
\end{abstract}

\section{$\S 1$. Introduction}

Let $f: M \rightarrow Y$ be a simple homotopy equivalence of closed $n$-manifolds, and let $X \subset Y$ be a submanifold of codimension $q$. The map $f$ splits along the submanifold $X \subset Y$ if there exists a map $g$ in the homotopy class of $f$ such that

(i) the map $g$ is transversal to $X$, so that, in particular, $g^{-1}(X)=N$ is a submanifold of $M$ of codimension $q$;

(ii) the restriction of the map $g$ to the submanifold $N$

$$
\left.g\right|_{N}: N \rightarrow X
$$

and the restriction of $g$ to $M \backslash N$

$$
\left.g\right|_{M \backslash N}:(M \backslash N) \rightarrow(Y \backslash X)
$$

are homotopy equivalences.

The research of the first author was carried out with the financial support of the President of the Russian Federation (grant no. 96-15-96841) and the Russian Foundation for Fundamental Research (grant no. 99-01-00009).

A part of the research was carried out at the Max-Plank-Institut für Mathematik in October, 1996.

AMS 1991 Mathematics Subject Classification. Primary 57R67, 57Q10, 19J25, 19G24; Secondary 57R10, 55U35, 18F25. 
There exist splitting obstruction groups $L S_{n-q}(F)$ that depend functorially on the push-out square

$$
F=\left(\begin{array}{ccc}
\pi_{1}(\partial U) & \longrightarrow & \pi_{1}(Y \backslash X) \\
\downarrow & & \downarrow \\
\pi_{1}(X) & \longrightarrow & \pi_{1}(Y)
\end{array}\right)
$$

of fundamental groups with orientation, where $U$ is a tubular neighbourhood of the submanifold $X$ in $Y$; these groups depend on the dimension $n-q \bmod 4$ (see [1] and [2]). For simplicity we shall denote the groups with orientation in the square $F$ as follows:

$$
F=\left(\begin{array}{lll}
A & \longrightarrow & C \\
\downarrow & & \downarrow \\
B & \longrightarrow & D
\end{array}\right) .
$$

Let $f: M \rightarrow Y$ be a normal map of degree 1 . Then there exists an obstruction to the existence of a map $g$ with properties (i), (ii) in the class of the normal cobordism of the map $f$. This obstruction lies in the group $L P_{n-q}(F)$, which also depends functorially on the square $F$ and depends on $n-q \bmod 4$.

The groups $L S_{*}(F)$ and $L P_{*}(F)$ are closely related to the $L$-groups and natural maps in the $L$-theory. For instance, these groups fit into the commutative diagram of exact sequences (see [1] and [2])

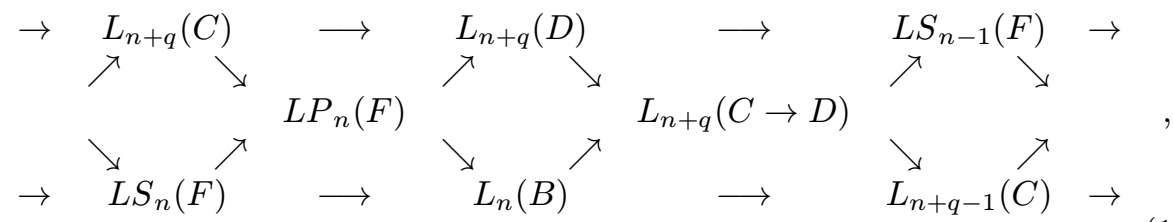

where almost all maps have a natural geometric description.

If $X$ is a one-sided submanifold of the manifold $Y$ and the horizontal maps in the square $F$ are isomorphisms, then the groups $L S_{*}(F)$ coincide with the BrowderLivesay groups $L N_{*}(C \rightarrow D)$. Such a pair of manifolds is called a Browder-Livesay pair. The groups $L P_{n}$ for a Browder-Livesay pair coincide with the relative groups $L_{n+1}\left(i^{!}\right)$, where $i^{!}: L_{n+1}(B) \rightarrow L_{n+1}(A)$ is the transfer map.

The Browder-Livesay groups are used in many geometric problems. For example, these groups are applied to the investigation of involutions on manifolds and to the problem of the realization of elements of the Wall groups by normal maps of closed manifolds (see [3]-[6]).

From the algebraic point of view the Browder-Livesay groups $L N_{*}(C \rightarrow D)$ are the $L$-groups of the ring with antistructure $(\mathbb{Z} C, \alpha, u)$, where $\alpha(x)=t \bar{x} t^{-1}$, $u=-w(t) t^{2}, t \in D \backslash C$, and the bar denotes the standard involution in the ring $\mathbb{Z} C: \Sigma n_{g} g \rightarrow \Sigma n_{g} w(g) g^{-1}$, where $w: C \rightarrow\{ \pm 1\}$ is the orientation homomorphism.

The case of a one-sided submanifold, when the horizontal maps in the square $F$ are epimorphisms, was considered in [7]-[10]. The square (1.1) is called in this case a geometric diagram [7], [8]. In particular, the groups $L S_{*}$ and $L P_{*}$ are defined for a square of antistructures (see [9] and [10]) in which the horizontal maps are epimorphisms and the vertical maps are quadratic extensions of antistructures [11]. 
A square of antistructures of this type can be obtained from the geometric diagram $F$ by a passage to the square of group rings with standard involutions.

It must be observed that in geometric applications we encounter only antistructures of a special type that arise as antistructures on the group rings of groups with additional structures.

A geometric antistructure [12], [13] is a group $\pi$ with an additional structure (see $\S 2$ ) that gives one the possibility of defining in a natural way an antistructure on the group ring $R \pi$. Only such antistructures are possible for the Wall surgery obstruction groups or the Browder-Livesay groups.

At present there exist effective methods for the computation of $L$-groups and $L N$ groups in the case of finite hyperelementary groups (see, for example, [6], [12]-[15]) and, in particular, in the case of finite 2-groups. These groups occupy a special place in surgery theory since the range of the map $\sigma:[M, G / T O P] \rightarrow L_{n}(G, w)$ in the Sullivan exact sequence is described by means of the $L$-group of the Sylow 2 -subgroup of $G$ [16]. The most complete results in computation were obtained for the projective $L$-groups (see [12] and [15]) introduced by Novikov [17].

In this paper we introduce the concept of geometric diagram of groups. This is a commutative square of groups equipped with geometric antistructures in which the horizontal maps are epimorphisms and the vertical maps are inclusions of index 2 . In addition, all maps of groups must be morphisms of geometric antistructures. The geometric diagram of groups is a natural generalization of the square $F$ arising in the splitting problem for a one-sided submanifold. For geometric diagrams of groups, using the methods of [9] and [10] we define the $L S$-groups and the $L P$-groups and describe their properties. In the case of finite 2-groups with antistructures we obtain fairly complete results, which are necessary for the computation of the $L S_{*}^{p}$ and $L P_{*}^{p}$-groups and the natural maps in the diagram (1.3). In fact, we reduce the computation of these groups and the natural maps to the computations for the $L_{*}^{p}$-groups carried out in [12].

\section{$\S 2$. A geometric diagram of groups}

First, we recall the necessary definitions.

Let $R$ be a ring with 1 . An antistructure is defined by a triple $(R, \alpha, u)$, where $\alpha$ is an anti-automorphism of the ring $R$ and $u \in R^{*}$ is a unit such that $\alpha(u)=u^{-1}$ and $\alpha^{2}(x)=u x u^{-1}$ for each $x \in R$. In this case the Wall groups $L_{n}^{X}(R, \alpha, u)$ are well defined for any subgroup $X \subset K_{i}(R), i=0,1$, that is invariant under the involution induced by $\alpha[18]-[20]$.

Let $R$ be the group ring $\mathbb{Z} \pi$ and let $V$ be the image of $\{ \pm \pi\}$ in $K_{1}(\mathbb{Z} \pi)$. The surgery obstruction groups $L_{n}(\pi, w)=L_{n}^{s}(\pi, w)$ are isomorphic to $L_{n}^{V}\left(\mathbb{Z} \pi,{ }^{-}, 1\right)$ in even dimensions, and one must take the quotients of the groups $L_{2 k+1}^{V}\left(\mathbb{Z} \pi,{ }^{-}, 1\right)$ by the subgroups $\mathbb{Z} / 2$ generated by the class of the automorphism $\sigma[18]$. We shall use similar agreements also for Browder-Livesay groups.

Let $(R, \alpha, u)$ be an antistructure with unit $v \in R^{*}$. There also exists an antistructure $\left(R, \beta, u_{1}\right)$ such that $u_{1}=v \alpha\left(v^{-1}\right) u$ and $\beta(x)=v \alpha(x) v^{-1}$ for each $x \in R$. Then one can consider the scaling isomorphism $L_{n}(R, \alpha, u) \cong L_{n}\left(R, \beta, u_{1}\right)$ induced by the multiplication of the quadratic form by $v$ on the left [18], [11].

A geometric antistructure [12], [13] on a group $\pi$ is a 4 -tuple $(\pi, w, \theta, b)$, where $w: \pi \rightarrow\{ \pm 1\}$ is an orientation homomorphism, $\theta \in \operatorname{Aut}(\pi), b \in \pi$, and the following 
conditions hold:

$$
\begin{aligned}
& \text { (i) } w \theta(g)=w(g) \text { for all } g \in \pi, \\
& \text { (ii) } \theta^{2}(g)=b g b^{-1} \text { for all } g \in \pi, \\
& \text { (iii) } \theta(b)=b \text { and } w(b)=1 .
\end{aligned}
$$

An orientation of a geometric antistructure is defined by an invertible element $\varepsilon \in R^{*}$ such that $\bar{\varepsilon}=\varepsilon^{-1}$, where the bar denotes an involution in the ring $R$.

The associated antistructure $(R \pi, \alpha, u)$ consists of the group ring $R \pi$ with antiautomorphism

$$
\alpha: \Sigma r_{g} g \rightarrow \Sigma \bar{r}_{g} w(g) \theta\left(g^{-1}\right)
$$

and unit $u=\varepsilon b$.

Let $(\pi, w, \theta, b, \varepsilon)$ be an oriented geometric antistructure and let $c \in \pi$. We can define another geometric antistructure $\left(\pi, w, \theta^{c}, b^{c}, \varepsilon^{c}\right)[13]$, where $\theta^{c}(x)=c \theta(x) c^{-1}$ for each $x \in \pi, b^{c}=c \theta(c) b$, and $\varepsilon^{c}=w(c) \varepsilon$. Then the antistructure associated with $\left(\pi, w, \theta^{c}, b^{c}, \varepsilon^{c}\right)$ is scaling-equivalent to the antistructure $(R \pi, \alpha, u)[13]$.

In what follows we shall consider the case when $\bar{r}=r(r \in R)$ and $\varepsilon= \pm 1$. Hence the oriented geometric antistructure $(\pi, \varepsilon)$ defines an antistructure on the ring $R \pi$.

In geometry, $L$-groups occur as surgery obstruction groups $L_{*}(\pi, w)$, where $\pi$ is the fundamental group of a Poincaré space endowed with an orientation homomorphism $w: \pi \rightarrow\{ \pm 1\}$. The $L$-groups of a ring with non-trivial antistructure occur in the splitting problem for Browder-Livesay pairs of manifolds $X \subset Y$. In this case the map $\pi_{1}(Y \backslash X) \rightarrow \pi_{1}(Y)$ in the square (1.1) is the inclusion of index 2 of groups with orientation. Moreover, an arbitrary inclusion $(\pi \rightarrow G, w)$ of index 2 of groups with orientation produces the Browder-Livesay groups

$$
L N_{*}(\pi \rightarrow G, w) \cong L_{*}\left(\mathbb{Z} \pi, \alpha,-w(t) t^{2}\right),
$$

where $t \in G \backslash \pi$ and $\alpha(x)=t \bar{x} t^{-1}$. The Browder-Livesay groups $L N_{n}(\pi \rightarrow G, w)$ coincide with the surgery obstruction groups $L_{n+1+w(t)}(\pi)$ if $\pi \subset G$ is a direct summand.

Remark 2.3. The antistructure in (2.2) depends on $t$. It is easy to verify that one can obtain another antistructure, $\left(\mathbb{Z} \pi, \beta,-w(\tau) \tau^{2}\right)$ with $\tau=v t, v \in \pi$, from the antistructure in (2.2) by means of scaling by $v$ (see, for example, [5]). To avoid functorial problems we shall identify each group $L_{*}\left(\mathbb{Z} \pi, \alpha,-w(t) t^{2}\right)$ with the subset of the direct product $\prod_{t \in G \backslash \pi} L_{*}\left(\mathbb{Z} \pi, \alpha,-w(t) t^{2}\right)$ consisting of elements whose components are connected by the scaling isomorphism (see [5]). The identification is given by the projection onto a coordinate plane. Hence $L$-groups depend on the class of scaling-equivalent antistructures.

An arbitrary geometric antistructure $(\pi, \theta, w, b)$ defines an inclusion $i: \pi \rightarrow G$ of index $2[12]$, where

$$
G=\left\{\pi * \mathbb{Z} / t g t^{-1}=\theta(g): t^{2}=b\right\} .
$$

Using the orientation of the geometric antistructure we can regard $i$ as a map $w: \pi \rightarrow\{ \pm 1\}$ of groups with orientation, $w(t)=-\varepsilon$. The inclusion $(\pi \rightarrow G, w)$ induces the short exact sequence

$$
1 \rightarrow \pi \rightarrow G \rightarrow \mathbb{Z} / 2 \rightarrow 1
$$


in which the map $\pi \rightarrow G$ agrees with the orientations. Thus, the oriented geometric antistructure $(\pi, \theta, w, b, \varepsilon)$ gives rise to the exact sequence (2.4), which we denote by $\mathcal{S}(\pi, \theta, w, b, \varepsilon)$.

The $L$-groups of the associated antistructure are isomorphic to the BrowderLivesay groups

$$
L_{*}(\mathbb{Z} \pi, \alpha, \varepsilon b) \cong L N_{*}(\pi \rightarrow G, w) .
$$

Example 2.5. Each group $\pi$ with orientation $w$ gives rise to the geometric antistructure $(\pi, w, \theta, b)$, where $\theta=\mathrm{Id}$ is the identity map, and $b=e$ is the unity element. In this case $G=\pi \oplus \mathbb{Z} / 2$.

Example 2.6. Let $\pi$ be the cyclic group $\mathbb{Z} / 2^{r+1}$ with generator $x$, let $b=x^{2^{r}}$, and let $\theta(x)=x^{-1}$. Then $i$ will be the standard inclusion of the cyclic group $\pi$ in the quaternion 2-group $G$.

A morphism of antistructures

$$
f:(R, \alpha, u) \rightarrow\left(R^{\prime}, \alpha^{\prime}, u^{\prime}\right)
$$

is defined by a ring homomorphism $f: R \rightarrow R^{\prime}$ such that $f(u)=u^{\prime}$ and $\alpha^{\prime} f=f \alpha$. We shall denote by $\mathcal{R} \mathcal{A}$ the category of classes of scaling-equivalent antistructures with these morphisms.

We define a morphism

$$
f:(\pi \rightarrow G, w) \longrightarrow\left(\pi^{\prime} \rightarrow G^{\prime}, w^{\prime}\right)
$$

of inclusions of index 2 of groups with orientation as a homomorphism $f: G \rightarrow G^{\prime}$ such that $w^{\prime} f=w$ and there exists a commutative diagram

$$
\begin{aligned}
& 1 \rightarrow \pi \rightarrow G \rightarrow \mathbb{Z} / 2 \rightarrow 1 \\
& 1 \rightarrow \begin{array}{l}
\downarrow \\
\pi^{\prime}
\end{array} \rightarrow \begin{array}{l}
\downarrow f \\
G^{\prime}
\end{array} \rightarrow \begin{array}{l}
\downarrow \cong \\
\mathbb{Z} / 2
\end{array} \rightarrow 1 .
\end{aligned}
$$

We denote by $\mathcal{G J}$ the category consisting of such exact sequences defined up to inner automorphisms, and such morphisms.

For an arbitrary ring $R$ the morphism $f$ in (2.7) induces a morphism of antistructures

$$
f_{a}:\left(R \pi, \alpha,-w(t) t^{2}\right) \rightarrow\left(R \pi^{\prime}, \alpha^{\prime},-w^{\prime}(f(t))(f(t))^{2}\right) .
$$

To see this it suffices to verify the condition $\alpha^{\prime} f_{a}=f_{a} \alpha$, where $\alpha$ and $\alpha^{\prime}$ are defined as in (2.2). Using our agreement in Remark 2.3 we obtain the functor

$$
\mathcal{H}: \mathcal{G J} \rightarrow \mathcal{R A}
$$

Let $(\pi, w, \theta, b)$ and $\left(\pi^{\prime}, w^{\prime}, \theta^{\prime}, b^{\prime}\right)$ be geometric antistructures with orientations $\varepsilon$ and $\varepsilon^{\prime}$, respectively. We define a morphism of oriented geometric antistructures

$$
f:(\pi, \varepsilon) \rightarrow\left(\pi^{\prime}, \varepsilon^{\prime}\right)
$$


as a homomorphism $f: \pi \rightarrow \pi^{\prime}$ such that

$$
\begin{aligned}
& \text { (i) } w^{\prime} f(g)=w(g) \text { for each } g \in \pi, \\
& \text { (ii) } \theta^{\prime} f(g)=f \theta(g) \text { for each } g \in \pi, \\
& \text { (iii) } f(b)=b^{\prime} \text { and } \varepsilon=\varepsilon^{\prime} \circ f \text {. }
\end{aligned}
$$

Let $\mathcal{G A}$ be the category of scaling-equivalent oriented geometric antistructures and these morphisms.

The map $f$ induces a map $\&(f)$ of inclusions of index 2 of groups with orientation $\mathcal{S}(\pi, \theta, w, b, \varepsilon) \rightarrow \mathcal{S}\left(\pi^{\prime}, \theta^{\prime}, w^{\prime}, b^{\prime}, \varepsilon^{\prime}\right)$. Thus, the map $\mathcal{S}$ is a functor

$$
\mathcal{S}: \mathcal{G A} \rightarrow \mathcal{G J}
$$

Under the assumptions (2.10) the map $f$ induces a morphism of the associated antistructures for the ring $R$ :

$$
\mathcal{F}(f):(R \pi, \alpha, \varepsilon b) \rightarrow\left(R \pi^{\prime}, \alpha^{\prime}, \varepsilon^{\prime} b^{\prime}\right),
$$

and therefore we obtain a functor $\mathcal{F}$ from the category of oriented geometric antistructures $\mathcal{G} \mathcal{A}$ to the category $\mathcal{R} \mathcal{A}$.

As is clear from the definitions, the following diagram is commutative:

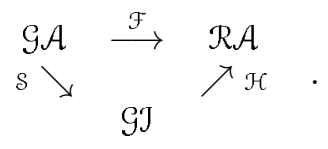

We consider now a morphism

$$
i:(\pi, w, \theta, b) \rightarrow\left(\pi^{\prime}, w^{\prime}, \theta^{\prime}, b^{\prime}\right)
$$

of geometric antistructures such that the map $i$ is an inclusion $\pi \rightarrow \pi^{\prime}$ of index 2 . We can identify the group $\pi$ in this case with a subgroup of $\pi^{\prime}, b$ with $b^{\prime}$, and $w$ with $w^{\prime}$. We shall denote such an inclusion of index 2 by $\left(\pi \rightarrow \pi^{\prime}, w, \theta, b\right)$, and we shall call it an inclusion of index 2 with geometric antistructure. We can equip this inclusion with an orientation $\varepsilon$.

Recall the definition of a quadratic extension of antistructures [11]. Let $(R, \alpha, u)$ be an antistructure and let $(\rho, a)$ be a structure on the ring $R$, that is, $\rho$ is an automorphism of $R, a \in R^{*}$, and we have $\rho(a)=a$ and $\rho^{2}(x)=a x a^{-1}$ for $x \in R$. The quadratic extension of an antistructure $(R, \alpha, u)$ with respect to the structure $(\rho, a)$ is the antistructure $(S, \alpha, u)$ such that $S=R[t] /\left(t^{2}-a\right), t x=\rho(x) t, \alpha(t) t \in R$, and $\alpha^{2}(t)=u t u^{-1}$.

We denote by $i$ the inclusion of antistructures

$$
(R, \alpha, u) \rightarrow(S, \alpha, u)
$$

and let $\gamma$ be the automorphism of the ring $S$ over $R$ defined by the formula $\gamma(x+y t)=(x-y t), x, y \in R$. The automorphism $\rho$ extends to the ring $S$ in accordance with the formula $\rho(x+y t)=t(x+y t) t^{-1}, x, y \in R$. There also exists 
another antistructure $(S, \widetilde{\alpha}, \widetilde{u})$ on the ring $S$, where $\widetilde{\alpha}=\rho \gamma \alpha$ and $\widetilde{u}=-t \alpha\left(t^{-1}\right) u$. Since $\widetilde{u} \in R$ and the ring $R$ is $\widetilde{\alpha}$-invariant, the antistructure $(R, \widetilde{\alpha}, \widetilde{u})$ is well defined. The quadratic extension of antistructures

$$
(R, \widetilde{\alpha}, \widetilde{u}) \rightarrow(S, \widetilde{\alpha}, \widetilde{u})
$$

coincides with the homomorphism $i$ as a map of rings.

Let $(\pi \rightarrow G, w, \theta, b, \varepsilon)$ be an inclusion of index 2 of oriented geometric antistructures. For each $t \in G \backslash \pi$ this inclusion defines a quadratic extension of the associated antistructures

$$
(R \pi, \alpha, u) \rightarrow(R G, \alpha, u)
$$

with respect to the structure $(\rho, a)$, where $u=\varepsilon b, \alpha\left(\Sigma r_{g} g\right)=\Sigma w(g) r_{g} \theta\left(g^{-1}\right)$, $a=t^{2}$, and $\rho\left(\Sigma r_{g} g\right)=\Sigma r_{g} t g t^{-1}, r_{g} \in R, g \in G$. To demonstrate this it is sufficient to verify the condition $\alpha(t) t \in R \pi$, which is obvious since $\theta(\pi)=\pi$. Hence the quadratic extension of antistructures

$$
(R \pi, \widetilde{\alpha}, \widetilde{u}) \rightarrow(R G, \widetilde{\alpha}, \widetilde{u})
$$

is well defined. The antistructure $(R \pi, \widetilde{\alpha}, \widetilde{u})$ depends on $t \in G \backslash \pi$.

Remark 2.12. Note that $\widetilde{u}=-\varepsilon w(t) t \theta(t) b$,

$$
\widetilde{\alpha}(t)=\rho \gamma \alpha(t)=-w(t) \rho \theta\left(t^{-1}\right)
$$

and the antiautomorphism $\widetilde{\alpha}$ on the ring $R \pi$ is defined by the formula

$$
\widetilde{\alpha}\left(\Sigma r_{x} x\right)=\Sigma r_{x} w(x) t \theta\left(x^{-1}\right) t^{-1}=\Sigma r_{x} w(x) \rho \theta\left(x^{-1}\right), \quad r_{x} \in R, \quad x \in \pi .
$$

Lemma 2.13. Let $\tau=v t(v \in \pi)$ be another element of $G \backslash \pi$. The antistructure $\left(R \pi, \widetilde{\alpha}^{\prime}, \widetilde{u}^{\prime}\right)$ obtained by the use of $\tau$ in place of $t$ is scaling-equivalent to the antistructure $(R \pi, \widetilde{\alpha}, \widetilde{u})$. The antistructure $\left(R G, \widetilde{\alpha}^{\prime}, \widetilde{u}^{\prime}\right)$ is scaling-equivalent to the antistructure $(R G, \widetilde{\alpha}, \widetilde{u})$.

Proof. Using Remark 2.12 we obtain

$$
\begin{aligned}
& \widetilde{\alpha}^{\prime}(x)=w(x)(v t) \theta\left(x^{-1}\right)(v t)^{-1}=v \widetilde{\alpha}(x) v^{-1} \quad \text { for each } x \in \pi, \\
& \widetilde{\alpha}^{\prime}(t)= \widetilde{\alpha}^{\prime}\left(v^{-1} v t\right)=\widetilde{\alpha}^{\prime}\left(v^{-1}\right) \widetilde{\alpha}^{\prime}(v t) \\
&= {\left[w\left(v^{-1}\right)(v t) \theta(v)(v t)^{-1}\right]\left[-w(v t)(v t) \theta\left((v t)^{-1}\right)(v t)^{-1}\right] } \\
&=-w(t) v t \theta\left(t^{-1}\right)(v t)^{-1}=v \widetilde{\alpha}(t) v^{-1}, \\
& \widetilde{u}^{\prime}=-\varepsilon w(v t) v t \theta(v t) b, \\
&-v \widetilde{\alpha}\left(v^{-1}\right) \widetilde{u}=v w(v) t \theta(v) t^{-1}(-\varepsilon) w(t) t \theta(t) b=-\varepsilon w(v t) v t \theta(v t) b .
\end{aligned}
$$

Hence, in view of the agreements in Remark 2.3, the class of the antistructure $(R \pi, \widetilde{\alpha}, \widetilde{u})$ is independent of $t \in G \backslash \pi$. 
Proposition 2.14. Let $(\pi \rightarrow G, w, \theta, b)$ be an inclusion of index 2 with orientation $\varepsilon$. Then there exists a geometric antistructure $(\pi, w, \widetilde{\theta}, \widetilde{b})$ with orientation $\widetilde{\varepsilon}$ such that

$$
\mathcal{F}(\pi, w, \widetilde{\theta}, \widetilde{b}, \widetilde{\varepsilon})=(R \pi, \widetilde{\alpha}, \widetilde{u}) .
$$

Proof. Let $t \in G \backslash \pi, \varepsilon^{\prime}=-w(t)$, and let $\rho$ be the automorphism of the group $\pi$ defined by the formula $\rho(x)=t x t^{-1}, x \in \pi$. Next, let

$$
\widetilde{\theta}=\rho \circ \theta, \quad \widetilde{b}=-w(t) \varepsilon^{\prime} t \theta(t) b=t \theta(t) b, \quad \widetilde{\varepsilon}=\varepsilon \varepsilon^{\prime}=-w(t) \varepsilon .
$$

We have

$$
\begin{gathered}
w \widetilde{\theta}(x)=w\left(t(\theta(x)) t^{-1}\right)=w \theta(x)=w(x), \\
\widetilde{\theta}^{2}(x)=(\rho \circ \theta)^{2}(x)=t\left[\theta\left(t(\theta(x)) t^{-1}\right)\right] t^{-1}=t \theta(t) \theta^{2}(x) \theta\left(t^{-1}\right) t^{-1} \\
=t \theta(t) b x b^{-1}(t \theta(t))^{-1}=t \theta(t) b x(t \theta(t) b)^{-1}=\widetilde{b} x \widetilde{b}^{-1} \quad \text { for each } x \in \pi, \\
w(\widetilde{b})=w\left(\left[-w(t) \varepsilon^{\prime}\right] t \theta(t) b\right)=w\left([-w(t)]^{2} t \theta(t) b\right)=w(b)=1, \text { since } w(\theta(t))=w(t),
\end{gathered}
$$

and

$$
\widetilde{\theta}(\widetilde{b})=t \theta[t \theta(t) b] t^{-1}=t \theta(t)\left[\theta^{2}(t)\right] \theta(b) t^{-1}=t \theta(t)\left[b t b^{-1}\right] b t^{-1}=t \theta(t) b=\widetilde{b} .
$$

Conditions (2.1) are now satisfied. For the associated antistructure we have

$$
\begin{gathered}
\widetilde{\varepsilon} \widetilde{b}=-w(t) \varepsilon t \theta(t) b=\widetilde{u} \\
w(g) \widetilde{\theta}\left(g^{-1}\right)=w(g) \rho \theta\left(g^{-1}\right)=\widetilde{\alpha}\left(g^{-1}\right), \quad g \in \pi .
\end{gathered}
$$

Proposition 2.16. Let $i=(\pi \rightarrow G, w, \theta, b)$ be an inclusion of index 2 of geometric antistructures with orientation $\varepsilon$. Then there exists an inclusion $\widetilde{i}=(\pi \rightarrow G, \widetilde{w}, \widetilde{\theta}, \widetilde{b})$ of index 2 of geometric antistructures with orientation $\widetilde{\varepsilon}$ such that $\mathcal{F}(\widetilde{i})$ is a quadratic extension $(R \pi, \widetilde{\alpha}, \widetilde{u}) \rightarrow(R G, \widetilde{\alpha}, \widetilde{u})$.

Proof. Let $\widetilde{w}(t)=-w(t),\left.\widetilde{w}\right|_{\pi}=w$, and let $\widetilde{\theta}, \widetilde{b}, \widetilde{\varepsilon}$ be defined by formula (2.15). Then $\widetilde{w}(t) \widetilde{\theta}\left(t^{-1}\right)=-w(t) \rho \theta\left(t^{-1}\right)=\widetilde{\alpha}(t)$ and the required result follows from Proposition 2.14 .

Proposition 2.17. Under the hypotheses of Proposition 2.16 the antistructures $\mathcal{F}(G, \widetilde{w}, \widetilde{\theta}, \widetilde{b}, \widetilde{\varepsilon})$ and $\mathcal{F}(G, \widetilde{w}, \theta, b, \varepsilon)$ are scaling equivalent. In particular, the corresponding L-groups are isomorphic.

Proof. Using the definition of the associated antistructure it is easy to verify that the required scaling is defined by the element $t^{-1}$. 
Example 2.18. We can consider each inclusion $(\pi \rightarrow G, w)$ of index 2 of groups with orientation as an inclusion $(\pi \rightarrow G, w, \mathrm{Id}, e)$ of index 2 equipped with a geometric antistructure with orientation $\varepsilon=1$. Then $\theta=\mathrm{Id}$, and we see from (2.15) that $\widetilde{\theta}(x)=t x t^{-1}, \widetilde{e}=t \theta(t) e=t^{2}$, and $\widetilde{\varepsilon}=-w(t) \varepsilon=-w(t)$ for $t \in G \backslash \pi$ and each $x \in \pi$. Hence $\mathcal{F}(\pi, w, \widetilde{\mathrm{Id}}, \widetilde{e}, \widetilde{1})=\left(R \pi, \beta,-w(t) t^{2}\right)$, where $\beta(x)=w(x) t x t^{-1}$ for each $x \in \pi$, and $\beta(r)=r$ for each $r \in R$. In the case when $R=\mathbb{Z}$ we have

$$
L_{n}(\mathcal{F}(\pi, w, \widetilde{\mathrm{Id}}, \widetilde{e}, \widetilde{1}))=L_{n}\left(\mathbb{Z} \pi, \beta,-w(t) t^{2}\right)=L N_{n}(\pi \rightarrow G, w) .
$$

Definition 2.19. A geometric diagram of groups is a commutative square of oriented geometric antistructures

$$
\begin{array}{ccc}
(\pi, w, \theta, b, \varepsilon) & \rightarrow & \left(\rho, w^{\prime}, \theta^{\prime}, b^{\prime}, \varepsilon^{\prime}\right) \\
\downarrow & & \downarrow \\
(G, w, \theta, b, \varepsilon) & \rightarrow & \left(H, w^{\prime}, \theta^{\prime}, b^{\prime}, \varepsilon^{\prime}\right)
\end{array}
$$

in which the vertical maps are inclusions of geometric antistructures of index 2 and the horizontal maps $\pi \rightarrow \rho, G \rightarrow H$ are epimorphisms.

Example 2.20. Let $F$ be the commutative diagram of groups (1.2) in the case of a one-sided submanifold. Note that all maps in the square $F$ agree with orientation except for the map $B \rightarrow D$. This map commutes with the orientation homomorphism in the range of the vertical map but not outside this range. For a study of the algebraic properties of $L$-functors on such squares we change the orientation on the group $B$ outside the range of the vertical map, which is an inclusion of index 2 . After such a change all homomorphisms in the square $F$ become compatible with orientation and we can regard the square $F$ as a geometric diagram of groups

$$
F=\left(\begin{array}{ccc}
(A, w, \operatorname{Id}, e, 1) & \stackrel{f}{\longrightarrow} & \left(C, w^{\prime}, \operatorname{Id}, e, 1\right) \\
\downarrow i & & \downarrow j \\
(B, w, \operatorname{Id}, e, 1) & \stackrel{g}{\longrightarrow} & \left(D, w^{\prime}, \operatorname{Id}, e, 1\right)
\end{array}\right) .
$$

Let $t \in B \backslash A, \tau=g(t) \in D \backslash C$. Then there exists a geometric diagram of groups

$$
\widetilde{F}=\left(\begin{array}{ccc}
\left(A, w, \rho, t^{2},-w(t)\right) & \stackrel{f}{\longrightarrow} & \left(C, w^{\prime}, \rho^{\prime}, \tau^{2},-w^{\prime}(\tau)\right) \\
\downarrow i & & \downarrow j \\
\left(B, w, \rho, t^{2},-w(t)\right) & \stackrel{g}{\longrightarrow} & \left(D, w^{\prime}, \rho^{\prime}, \tau^{2},-w^{\prime}(\tau)\right)
\end{array}\right),
$$

where $\rho(x)=\widetilde{\operatorname{Id}}(x)=t x t^{-1}$ for each $x \in B$ and $\rho^{\prime}(y)=\widetilde{\operatorname{Id}}(y)=\tau y \tau^{-1}$ for each $y \in D$.

For $R=\mathbb{Z}$ the square of antistructures $\mathcal{F}(\widetilde{F})$ induces the square of $L$-groups

$$
\begin{array}{ccc}
L N_{n}(A \rightarrow B, w) & \longrightarrow & L N_{n}\left(C \rightarrow D, w^{\prime}\right) \\
\downarrow & & \downarrow \\
L_{n}\left(B^{-}\right) & \longrightarrow & L_{n}\left(D^{-}\right)
\end{array},
$$

which is obtained by using the isomorphism (2.2) and scaling equivalences. Here the superscript "-" signifies the change of orientation on the elements $t$ and $\tau$.

Remark 2.24. In [9] and [10] the term 'geometric diagram of groups' is used only for diagrams (1.1) in which the horizontal maps are epimorphisms and the vertical maps are inclusions of index 2 . 


\section{$\S 3$. $L S$ - and $L P$-groups of geometric diagrams of groups}

In this section we define $L S$ - and $L P$-groups and describe their properties in the case of geometric diagrams of groups

$$
F=\left(\begin{array}{ccc}
(\pi, w, \theta, b, \varepsilon) & \rightarrow & \left(\rho, w^{\prime}, \theta^{\prime}, b^{\prime}, \varepsilon^{\prime}\right) \\
\downarrow & & \downarrow \\
(G, w, \theta, b, \varepsilon) & \rightarrow & \left(H, w^{\prime}, \theta^{\prime}, b^{\prime}, \varepsilon^{\prime}\right)
\end{array}\right)=\left(\begin{array}{cccc}
\pi & \stackrel{f}{\longrightarrow} & \rho \\
\downarrow i & & \downarrow j \\
G & \stackrel{g}{\longrightarrow} & H
\end{array}\right) .
$$

In what follows we shall sometimes write $\pi$ instead of $(\pi, w, \theta, b, \varepsilon)$ if this does not lead to confusion. We shall use similar agreements for maps and squares of geometric antistructures.

For a ring $T$ the square $F$ defines the geometric diagram of antistructures [9]

$$
\Phi=\mathcal{F}(F)=\left(\begin{array}{ccc}
(R, \alpha, u) & \stackrel{f}{\longrightarrow} & (P, \beta, v) \\
\downarrow i & & \downarrow j \\
(S, \alpha, u) & \stackrel{g}{\longrightarrow} & (Q, \beta, v)
\end{array}\right)=\left(\begin{array}{ccc}
R & \rightarrow & P \\
\downarrow & & \downarrow \\
S & \rightarrow & Q
\end{array}\right),
$$

where $R=T \pi, P=T \rho, S=T G$, and $Q=T H$ are rings with associated antistructures. We shall denote the induced map of the associated antistructures in the same way as the corresponding map of geometric antistructures. The vertical maps in the square (3.2) are quadratic extensions of antistructures and the horizontal maps are epimorphisms of antistructures.

Hence (see [9] and [10]) there exists a geometric diagram of antistructures

$$
\widetilde{\Phi}=\left(\begin{array}{ccc}
(R, \widetilde{\alpha}, \widetilde{u}) & \stackrel{\tilde{f}}{\longrightarrow} & (P, \widetilde{\beta}, \widetilde{v}) \\
\downarrow \widetilde{i} & & \downarrow \widetilde{j} \\
(S, \widetilde{\alpha}, \widetilde{u}) & \stackrel{\tilde{g}}{\longrightarrow} & (Q, \widetilde{\beta}, \widetilde{v})
\end{array}\right)=\left(\begin{array}{ccc}
\widetilde{R} & \rightarrow & \widetilde{P} \\
\downarrow & & \downarrow \\
\widetilde{S} & \rightarrow & \widetilde{Q}
\end{array}\right) .
$$

Consider now the geometric diagram of groups

$$
\widetilde{F}=\left(\begin{array}{ccc}
(\pi, \widetilde{w}, \widetilde{\theta}, \widetilde{b}, \widetilde{\varepsilon}) & \rightarrow & \left(\rho, \widetilde{w}^{\prime}, \widetilde{\theta}^{\prime}, \widetilde{b}^{\prime}, \widetilde{\varepsilon}^{\prime}\right) \\
\downarrow & & \downarrow \\
(G, \widetilde{w}, \widetilde{\theta}, \widetilde{b}, \widetilde{\varepsilon}) & \rightarrow & \left(H, \widetilde{w}^{\prime}, \widetilde{\theta}^{\prime}, \widetilde{b}^{\prime}, \widetilde{\varepsilon}^{\prime}\right)
\end{array}\right)=\left(\begin{array}{ccc}
\widetilde{\pi} & \stackrel{\widetilde{f}}{\longrightarrow} & \widetilde{\rho} \\
\downarrow \widetilde{i} & & \downarrow \widetilde{j} \\
\widetilde{G} & \stackrel{\widetilde{g}}{\longrightarrow} & \widetilde{H}
\end{array}\right)
$$

such that $\mathcal{F}(\widetilde{F})=\widetilde{\Phi}$. The existence of this diagram follows from Propositions 2.14 and 2.16 .

Now let $T$ be a fixed ring with 1 . Following [12] and [13] we define the $L$-groups of a geometric antistructure as the $L$-groups of the associated antistructure. The relative $L$-groups are defined in a similar way. For example, we have

$$
L_{*}(\pi)=L_{*}(\pi, w, \theta, b, \varepsilon)=L_{*}(R, \alpha, u), \quad L_{*}(\widetilde{f})=L_{*}(\widetilde{R} \rightarrow \widetilde{P}) .
$$

Let $G^{-}$be the geometric antistructure $(G, \widetilde{w}, \theta, b, \varepsilon)$, and let $H^{-}$be the geometric antistructure $\left(H, \widetilde{w}^{\prime}, \theta^{\prime}, b^{\prime}, \varepsilon^{\prime}\right)$. Then we have the quadratic extensions of antistructures $(\pi \rightarrow G, \widetilde{w}, \theta, b, \varepsilon)=\left(\pi \rightarrow G^{-}\right)$and $\left(\rho \rightarrow H, \widetilde{w}^{\prime}, \theta^{\prime}, b^{\prime}, \varepsilon^{\prime}\right)=\left(\rho \rightarrow H^{-}\right)$. We denote these extensions by $i_{-}$and $j_{-}$, respectively. 
From Proposition 2.17 we obtain the isomorphisms

$$
L_{*}(\widetilde{G}) \cong L_{*}\left(G^{-}\right), L_{*}(\widetilde{H}) \cong L_{*}\left(H^{-}\right), \quad L_{*}(\widetilde{g}) \cong L_{*}\left(g^{-}\right),
$$

where $g^{-}$is the morphism $G^{-} \rightarrow H^{-}$induced by $g$.

There exist relative $L$-groups $L_{*}\left(i^{!}\right), L_{*}\left(i_{-}^{!}\right), L_{*}\left(j^{!}\right)$, and $L_{*}\left(j_{-}^{!}\right)$fitting into the corresponding exact sequences for transfer maps. We shall denote by $L_{*}\left(\left(i_{-}, j_{-}\right)^{!}\right)=$ $L_{*}\left(F_{-}^{!}\right)$the relative groups fitting into the exact sequence

$$
\rightarrow L_{*}\left(g^{-}\right) \rightarrow L_{*}(f) \rightarrow L_{*}\left(F_{-}^{!}\right) \rightarrow
$$

for the transfer map of relative $L$-groups.

In [9] and [10] the $L S_{*^{-}}$and $L P_{*}$-groups are defined for an arbitrary geometric diagram of antistructures. Hence we can define the $L S_{*}$ - and $L P_{*}$-groups for a geometric diagram of groups $F$ as the corresponding groups for the geometric diagram of antistructures $\Phi=\mathcal{F}(F)$ :

$$
L S_{n}(F) \stackrel{\text { def }}{=} L S_{n}(\Phi), \quad L P_{n}(F) \stackrel{\text { def }}{=} L P_{n}(\Phi) \quad \text { for all } n=0,1,2,3 \bmod 4 .
$$

In particular, we shall denote by $L N_{*}(\pi \rightarrow G, w, \theta, b, \varepsilon)$ the $L S_{*}$-groups of the geometric diagram

$$
F=\left(\begin{array}{ccc}
(\pi, w, \theta, b, \varepsilon) & \rightarrow & (\pi, w, \theta, b, \varepsilon) \\
\downarrow & & \downarrow \\
(G, w, \theta, b, \varepsilon) & \rightarrow & (G, w, \theta, b, \varepsilon)
\end{array}\right),
$$

in which the horizontal maps are the identity maps.

Let us recall the properties of the $L S$ - and $L P$-groups of geometric diagrams. The results here are reformulations of results known for a geometric diagram of antistructures (see [1], [2], [6], [9], and [10]).

Theorem 3.7. Let $F$ be the geometric diagram of groups (3.1). Then there exist commutative diagrams of exact sequences

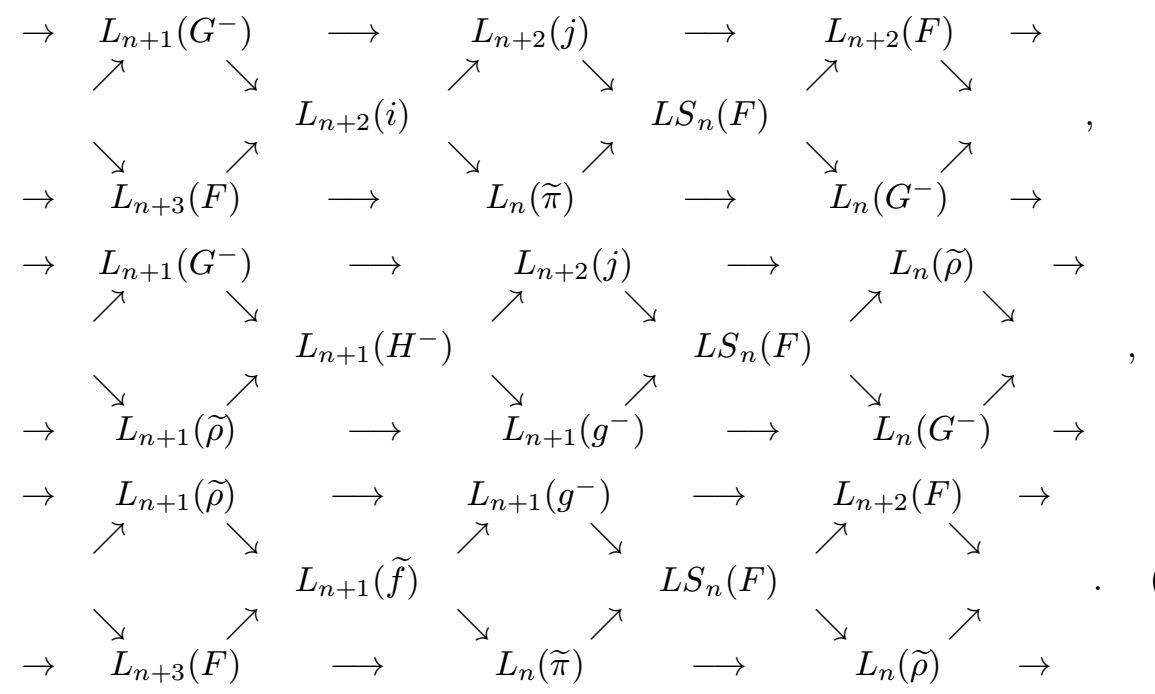


Example 3.11. Let $F$ be the geometric diagram (3.6). Then from the diagram (3.8) we see that $L N_{*}(\pi \rightarrow G)=L S_{*}(F) \cong L_{*}(\widetilde{\pi})$.

Theorem 3.12. Under the hypotheses of Theorem 3.7 there exist the following diagrams of exact sequences:

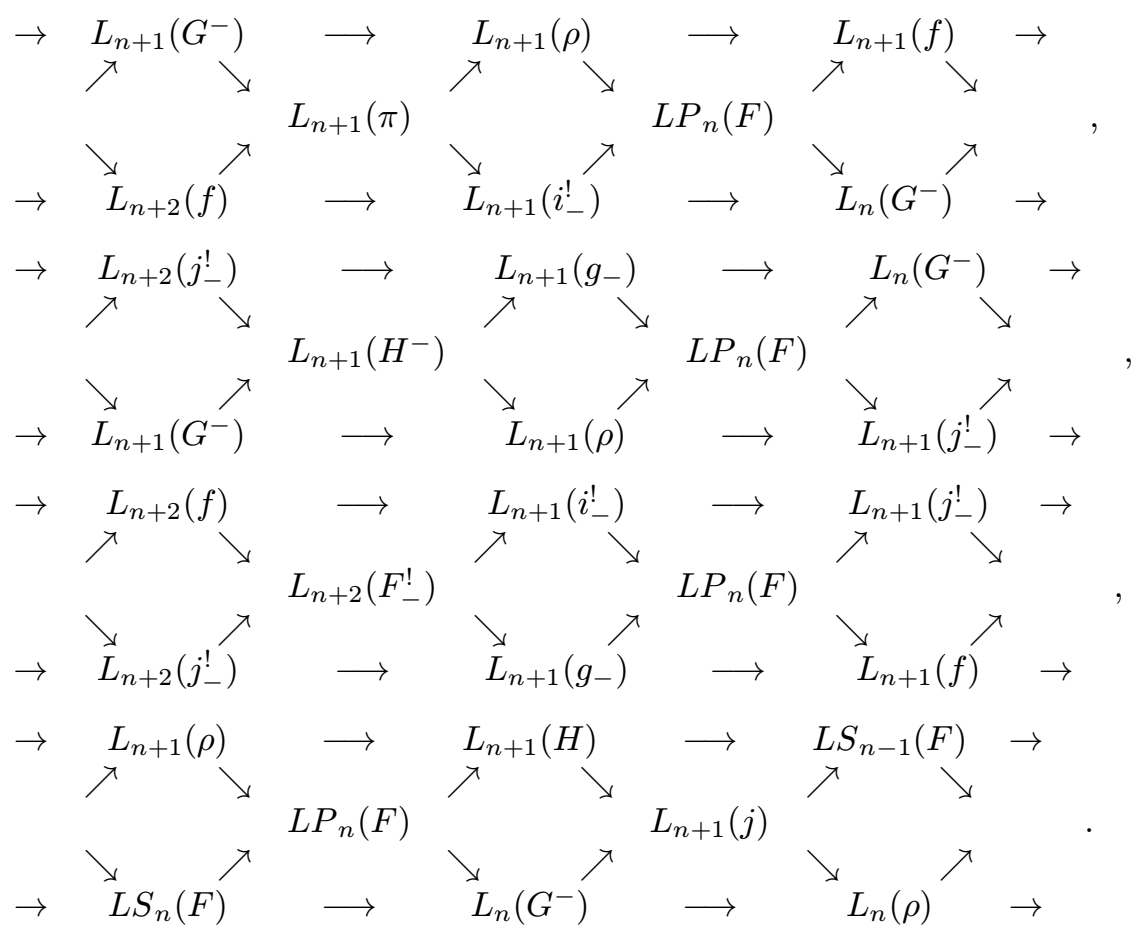

The diagram (3.16) is in fact the diagram (1.3) in the case when (1.2) is a geometric diagram of groups.

Example 3.17. Let $F$ be the geometric diagram (3.6). Then it follows from the diagram (3.15) that $L P_{*}(F) \cong L_{*+1}\left(i_{-}^{!}\right) \cong L_{*+1}\left(j_{-}^{!}\right)$.

Remark 3.18. In view of the agreements of $\S 2$ we can regard all groups in this section as groups decorated by " $s$ ". Similar results hold also in the cases of $L$-groups decorated by " $p$ " or " $h$ ".

\section{§ 4. $L S^{p}$ - and $L P^{p}$-groups for finite 2-groups}

In this section we shall consider only the case of groups decorated by " $p$ ". In what follows $L S_{*}$ means $L S_{*}^{p}, L P_{*}$ means $L P_{*}^{p}$, and a similar agreement holds for $L$-groups if this does not lead to confusion. In the rest we preserve the notation of $\S 3$ in the case $T=\mathbb{Z}$; for example,

$$
L_{*}(\pi)=L_{*}^{p}(\pi, w, \theta, b, \varepsilon)=L_{*}^{p}(\mathbb{Z} \pi, \alpha, u),
$$

where $(\mathbb{Z} \pi, \alpha, u)$ is the associated antistructure. 
In our notation for $L_{*}^{p}, L S_{*}^{p}$ and $L P_{*}^{p}$-groups we shall attach a subscript 2 to objects if $T=\widehat{\mathbb{Z}}_{2}$. For example,

$$
L_{*}\left(\pi_{2}\right)=L_{*}^{p}\left(\pi_{2}\right)=L_{*}^{p}\left(\widehat{\mathbb{Z}}_{2} \pi, \alpha, u\right),
$$

where $\left(\widehat{\mathbb{Z}}_{2} \pi, \alpha, u\right)$ is the associated antistructure.

We shall denote by $L_{*}^{\text {rel }}(\pi)$ the relative $L$-group $L_{*}\left(\mathbb{Z} \pi \rightarrow \widehat{\mathbb{Z}}_{2} \pi, \alpha, u\right)$ for the geometric antistructure $\pi=(\pi, w, \theta, b, \varepsilon)$. We shall use similar notation for $L S_{*}$, $L P_{*}, L N_{*}$, and relative $L$-groups. Thus, we have the long exact sequences

$$
\begin{aligned}
& \rightarrow L L_{*}(\pi) \rightarrow L_{*}\left(\pi_{2}\right) \rightarrow L_{*}^{\mathrm{rel}}(\pi) \rightarrow, \\
& \rightarrow L P_{*}(F) \rightarrow L P_{*}\left(F_{2}\right) \rightarrow L P_{*}^{\mathrm{rel}}(F) \rightarrow, \\
& \rightarrow L S_{*}(F) \rightarrow L S_{*}\left(F_{2}\right) \rightarrow L S_{*}^{\mathrm{rel}}(F) \rightarrow .
\end{aligned}
$$

Theorem 4.4. Theorems 3.7 and 3.12 hold in the case of groups equipped with superscript "rel".

Proof. The relative versions of these diagrams are obtained by standard methods using $L$-spectra (see [20] and [21]).

Assume now that the groups $\pi, \rho, G$ and $H$ in the geometric diagram (3.1) are finite 2 -groups. We start with a description of $L S_{*}\left(F_{2}\right)$ and $L P_{*}\left(F_{2}\right)$-groups and some natural maps in the case $T=\widehat{\mathbb{Z}}_{2}$.

Theorem 4.5 [12]. For a geometric antistructure on a finite 2-group $\pi$ there is an isomorphism

$$
L_{n}\left(\pi_{2}\right)=L_{n}^{p}\left(\widehat{\mathbb{Z}}_{2} \pi, \alpha, u\right) \stackrel{\cong}{\longrightarrow} L_{n}^{p}(\mathbb{Z} / 2, \mathrm{Id}, 1)= \begin{cases}\mathbb{Z} / 2, & n=0 \bmod 2 \\ 0, & n=1 \bmod 2 .\end{cases}
$$

Lemma 4.6. Let $f$ be an arbitrary morphism $(\pi, w, \theta, b, \varepsilon) \rightarrow\left(\rho, w^{\prime}, \theta^{\prime}, b^{\prime}, \varepsilon^{\prime}\right)$ of geometric antistructures. Then the induced map $f_{*}: L_{n}^{p}\left(\pi_{2}\right) \rightarrow L_{n}^{p}\left(\rho_{2}\right)$ is an isomorphism for each $n=0,1,2,3 \bmod 4$.

In particular,

$$
L_{n}^{p}\left(F_{2}\right)=L_{n}^{p}\left(f_{2}\right)=L_{n}^{p}\left(g_{2}\right)=L_{n}^{p}\left(j_{2}\right)=L_{n}^{p}\left(i_{2}\right)=0
$$

for the geometric diagram (3.1).

Proof. See [12]. In fact, the result follows from the commutative diagram

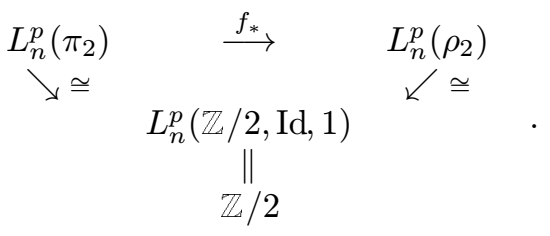


Theorem 4.7. Let $\pi, \rho, G$ and $H$ in the geometric diagram (3.1) be finite 2-groups. Then

$$
L S_{n}^{p}\left(F_{2}\right)= \begin{cases}\mathbb{Z} / 2, & n=0 \bmod 2, \\ 0, & n=1 \bmod 2 .\end{cases}
$$

Proof. Consider the long exact sequence

$$
\rightarrow L_{n+1}\left(g_{2}^{-}\right) \rightarrow L S_{n}\left(F_{2}\right) \rightarrow L_{n}(\widetilde{\rho}) \rightarrow
$$

from the diagram (3.10), where $g_{2}^{-}$is a morphism of geometric antistructures. The required result now follows from Lemma 4.6.

Lemma 4.8. Let $i:(\pi, w, \theta, b, \varepsilon) \rightarrow(G, w, \theta, b, \varepsilon)$ be an inclusion of index 2 of geometric antistructures. Then the transfer maps $\left(i^{!}\right): L_{n}^{p}\left(G_{2}\right) \rightarrow L_{n}^{p}\left(\pi_{2}\right)$ and $\left(i_{-}{ }^{!}\right): L_{n}^{p}\left(G_{2}^{-}\right) \rightarrow L_{n}^{p}\left(\pi_{2}\right)$ are trivial and

$$
L_{n}^{p}\left(\left(i_{ \pm}^{!}\right)_{2}\right)=\mathbb{Z} / 2 \quad \text { for all } n=0,1,2,3 \bmod 4 .
$$

Proof. Consider the diagram (3.16) for the square (3.6) in which the vertical maps are equal to the map $i$ :

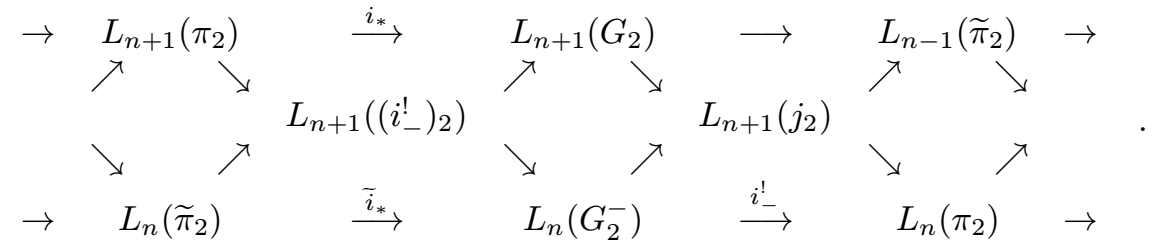

The rows in this diagram are chain complexes. The triviality of the map $i_{-}^{!}$(see [12]) now follows from Lemma 4.6. Hence the relative sequence for the map $i_{-}^{!}$gives rise to the short exact sequences

$$
0 \rightarrow L_{n}\left(\pi_{2}\right) \rightarrow L_{n}\left(\left(i_{-}^{!}\right)_{2}\right) \rightarrow L_{n-1}\left(G_{2}\right) \rightarrow 0 \quad \text { for all } n=0,1,2,3 \bmod 4,
$$

and the result follows by Theorem 4.5. The result concerning the map $i$ follows by a similar diagram constructed for the morphism $\pi \rightarrow G^{-}$of geometric antistructures.

Theorem 4.10. Under the hypotheses of Theorem 4.7,

$$
L P_{n}^{p}\left(F_{2}\right)=\mathbb{Z} / 2 \quad \text { for all } n=0,1,2,3 \bmod 4 .
$$

Proof. One must consider the long exact sequence

$$
\rightarrow L_{n+2}\left(f_{2}\right) \rightarrow L_{n+1}\left(\left(i_{-}^{!}\right)_{2}\right) \rightarrow L P_{n}\left(F_{2}\right) \rightarrow
$$

from the diagram (3.15), where $L_{n+2}\left(f_{2}\right)=0$ by Lemma 4.6.

We shall now describe the relative terms in the exact sequences (4.1) and (4.2). We recall the necessary facts from [12]. 
We have a decomposition of the group ring $\mathbb{Q} \pi$ into a direct sum of simple algebras:

$$
\mathbb{Q} \pi=\prod A_{\phi}
$$

where $A_{\phi}=a_{\phi}(\mathbb{Q} \pi)$ for some central idempotent $a_{\phi}$. We have $1=\sum a_{\phi}$, and no idempotent $a_{\phi}$ can be expressed as a sum of non-trivial central idempotents. Let $\alpha$ be the anti-automorphism of $\mathbb{Q} \pi$ induced by the geometric antistructure $(\pi, w, \theta, b, \varepsilon)$, and let $a_{\alpha(\phi)}$ be the idempotent $\alpha\left(a_{\phi}\right)$. This gives us a decomposition of the associated antistructure:

$$
(\mathbb{Q} \pi, \alpha, u)=\prod_{\phi=\alpha(\phi)}\left(A_{\phi}, \alpha_{\phi}, u_{\phi}\right) \times \prod_{\phi \cong \alpha(\phi)}\left(A_{\phi} \times A_{\alpha(\phi)}, \alpha_{\phi \times \alpha(\phi)}, u_{\phi} \times u_{\alpha(\phi)}\right)
$$

since $\alpha^{2}$ is an inner automorphism. The product for $\phi \cong \alpha(\phi)$ has the type $G L$ (a product of simple algebras interchanged by the antiautomorphism $\alpha$ ) and makes no contribution to $L$-groups. We have a similar decomposition for the antistructures $(\mathbb{Z}[1 / 2] \pi, \alpha, u)$ and $\left(\widehat{\mathbb{Q}}_{2} \pi, \alpha, u\right)$. Let

$$
\begin{aligned}
& (\mathbb{Z}[1 / 2] \pi, \alpha, u)=\prod_{\phi=\alpha(\phi)}\left(\Lambda_{\phi}, \alpha_{\phi}, u_{\phi}\right) \times \prod_{\phi \cong \alpha(\phi)}\left(\Lambda_{\phi} \times \Lambda_{\alpha(\phi)}, \alpha_{\phi \times \alpha(\phi)}, u_{\phi} \times u_{\alpha(\phi)}\right), \\
& \left(\widehat{\mathbb{Q}}_{2} \pi, \alpha, u\right)=\prod_{\phi=\alpha(\phi)}\left(\widehat{\Lambda}_{\phi 2}, \alpha_{\phi}, u_{\phi}\right) \times \prod_{\phi \cong \alpha(\phi)}\left(\widehat{\Lambda}_{\phi_{2}} \times \widehat{\Lambda_{\alpha(\phi)}}, \alpha_{\phi \times \alpha(\phi)}, u_{\phi} \times u_{\alpha(\phi)}\right) .
\end{aligned}
$$

Theorem $4.12[12]$. For each geometric antistructure $(\pi, w, \theta, b, \varepsilon)$ there is the following decomposition of the relative L-groups of the associated antistructure:

$$
L_{n}^{p}\left(\mathbb{Z} \pi \rightarrow \widehat{\mathbb{Z}}_{2} \pi, \alpha, u\right) \stackrel{\cong}{\rightarrow} L_{n}^{K}\left(\mathbb{Z}[1 / 2] \pi \rightarrow \widehat{\mathbb{Q}}_{2} \pi, \alpha, u\right) \stackrel{\cong}{\rightarrow} \prod_{\phi=\alpha(\phi)} L_{n}^{K_{1}}\left(\Lambda_{\phi} \rightarrow \widehat{\Lambda}_{\phi_{2}}, \alpha_{\phi}, u_{\phi}\right) .
$$

Consider an epimorphism $g:(G, w, \theta, b, \varepsilon) \rightarrow\left(H, w^{\prime}, \theta^{\prime}, b^{\prime}, \varepsilon^{\prime}\right)$ of geometric antistructures with finite 2-groups $G$ and $H$. Let $g$ be the induced epimorphism of the associated antistructures:

$$
\begin{aligned}
& \mathbb{Q} G=\underbrace{\prod_{\phi=\alpha(\phi)}\left(A_{\phi}, \alpha_{\phi}, u_{\phi}\right) \times \prod_{\phi \cong \alpha(\phi)}\left(A_{\phi} \times A_{\alpha(\phi)}, \alpha_{\phi \times \alpha(\phi)}, u_{\phi} \times u_{\alpha(\phi)}\right)}_{\prod_{\prod^{\prime}=\alpha\left(\phi^{\prime}\right)}\left(A_{\phi^{\prime}}, \alpha_{\phi^{\prime}}, u_{\phi^{\prime}}\right) \times \prod_{\phi^{\prime} \cong \alpha\left(\phi^{\prime}\right)}\left(A_{\phi^{\prime}} \times A_{\alpha\left(\phi^{\prime}\right)}, \alpha_{\phi^{\prime} \times \alpha\left(\phi^{\prime}\right)}, u_{\phi^{\prime}} \times u_{\alpha\left(\phi^{\prime}\right)}\right)} \\
& \mathbb{Q} H=
\end{aligned}
$$

The kernel of the epimorphism $g$ is a two-sided ideal $I$ in the ring $\mathbb{Q} G$, and the map $\mathbb{Q} G / I \rightarrow \mathbb{Q} H$ is an isomorphism. Since this ring is semisimple, the ideal $I$ is a direct product of some simple algebras $\prod_{1} A_{\phi}$ in (4.13). We can now write the decomposition (4.13) of the algebra $\mathbb{Q} G$ in the following form:

$$
\mathbb{Q} G=\prod_{1} A_{\phi_{1}} \times \prod_{2} A_{\phi_{2}}=P_{1} \times P_{2},
$$

where $\left.g\right|_{\prod_{2}}=\left.g\right|_{P_{2}}$ is an isomorphism. The map $\left.g\right|_{P_{2}}$ is an isomorphism of antistructures since $g: \mathbb{Q} G \rightarrow \mathbb{Q} H$ is a morphism of antistructures. Hence the map $g: \mathbb{Q} G \rightarrow \mathbb{Q} H$ is a projection of antistructures:

$$
g: P_{1} \times P_{2} \rightarrow P_{2} .
$$


Lemma 4.15. The epimorphism $g$ of geometric antistructures induces splitting projections $g_{*}: L_{n}^{\mathrm{rel}}(G) \rightarrow L_{n}^{\mathrm{rel}}(H)$ for each $n=0,1,2,3 \bmod 4$, and

$$
L_{n}^{\mathrm{rel}}(G) \cong L_{n+1}^{\mathrm{rel}}(g) \oplus L_{n}^{\mathrm{rel}}(H) .
$$

Proof. By Theorem 4.12 we have the isomorphisms

$$
L_{n}^{\mathrm{rel}}(G)=L_{n}^{p}\left(\mathbb{Z} G \rightarrow \widehat{\mathbb{Z}}_{2} G, \alpha, u\right) \stackrel{\cong}{\rightrightarrows} L_{n}^{K}\left(\mathbb{Z}[1 / 2] G \rightarrow \widehat{\mathbb{Q}}_{2} G, \alpha, u\right),
$$

and

$$
L_{n}^{\mathrm{rel}}(H)=L_{n}^{p}\left(\mathbb{Z} H \rightarrow \widehat{\mathbb{Z}}_{2} H, \alpha, u\right) \stackrel{\cong}{\rightrightarrows} L_{n}^{K}\left(\mathbb{Z}[1 / 2] H \rightarrow \widehat{\mathbb{Q}}_{2} H, \alpha, u\right) .
$$

Consider the commutative square of antistructures

$$
\begin{array}{ccc}
(\mathbb{Z}[1 / 2] G, \alpha, u) & \rightarrow & \left(\widehat{\mathbb{Q}}_{2} G, \alpha, u\right) \\
\downarrow & & \downarrow \\
\left(\mathbb{Z}[1 / 2] H, \alpha^{\prime}, u^{\prime}\right) & \rightarrow & \left(\widehat{\mathbb{Q}}_{2} H, \alpha^{\prime}, u^{\prime}\right)
\end{array},
$$

in which the vertical maps are induced by $g$. As follows from the decomposition (4.14) we can also write this square as follows:

$$
\begin{array}{ccc}
R \times S & \rightarrow & \widehat{R}_{2} \times \widehat{S}_{2} \\
\downarrow p & & \downarrow q \\
R & \rightarrow & \widehat{R}_{2}
\end{array}
$$

where

$$
\begin{aligned}
R \times S & =(\mathbb{Z}[1 / 2] G, \alpha, u), \quad \widehat{R}_{2} \times \widehat{S}_{2}=\left(\widehat{\mathbb{Q}}_{2} G, \alpha, u\right), \\
\widehat{R}_{2} & =\left(\widehat{\mathbb{Q}}_{2} H, \alpha^{\prime}, u^{\prime}\right), \quad R=\left(\mathbb{Z}[1 / 2] H, \alpha^{\prime}, u^{\prime}\right) .
\end{aligned}
$$

The vertical maps here are the natural projections, while the top map is the direct product of the maps $R \rightarrow \widehat{R}_{2}$ and $S \rightarrow \widehat{S}_{2}$.

Consider now the commutative diagram

$$
\begin{aligned}
& \rightarrow \begin{array}{ccccc}
L_{n}^{K}(R) & \rightarrow & L_{n}^{K}\left(\widehat{R}_{2}\right) & \rightarrow & L_{n}^{\mathrm{rel}}(H) \rightarrow \\
& \downarrow \varphi & \downarrow \psi & & \downarrow \chi
\end{array} \\
& \rightarrow \begin{array}{cccc}
L_{n}^{K}(R \times S) \\
\downarrow p_{*}
\end{array} \rightarrow \begin{array}{ccc}
L_{n}^{K}\left(\widehat{R}_{2} \times \widehat{S}_{2}\right) \\
\downarrow q_{*}
\end{array} \quad \rightarrow \begin{array}{c}
L_{n}^{\mathrm{rel}}(G) \\
\downarrow g_{*}
\end{array} \quad \rightarrow \quad, \\
& \rightarrow \quad L_{n}^{K}(R) \quad \rightarrow \quad L_{n}^{K}\left(\widehat{R}_{2}\right) \quad \rightarrow \quad L_{n}^{\mathrm{rel}}(H) \quad \rightarrow
\end{aligned}
$$

where the superscript $K$ means $K_{1}$. Here the top vertical maps $\varphi, \psi$, and $\chi$ are the Morita maps in round $L$-theory (see [20], Example 5.12). The map $\chi$ exists since the corresponding triad of $L$-groups is defined. The maps $\varphi$ and $\psi$ split the maps $p_{*}$ and $q_{*}$ [20], that is, $p_{*} \circ \varphi$ and $q_{*} \circ \psi$ are isomorphisms. Hence the composite $g_{*} \circ \chi$ is an isomorphism and the proof of the theorem is complete.

Remark 4.16. The results of [12] provide a full description of the relative groups $L_{n}^{\mathrm{rel}}(G)$ and $L_{n}^{\mathrm{rel}}(H)$ in the case under consideration. Thus, Lemma 4.15 gives one the groups $L_{*}^{\text {rel }}(g)$ for each epimorphism $g: G \rightarrow H$ of antistructures. 
Theorem 4.17. Under the hypotheses of Theorem 4.7 there exist natural isomorphisms

$$
L S_{n}^{\mathrm{rel}}(F) \cong L_{n+1}^{\mathrm{rel}}\left(g^{-}\right) \oplus L_{n}^{\mathrm{rel}}(\widetilde{\rho}) \quad \text { for all } n=0,1,2,3 \bmod 4 .
$$

Proof. Consider the diagram (3.10) for relative groups:

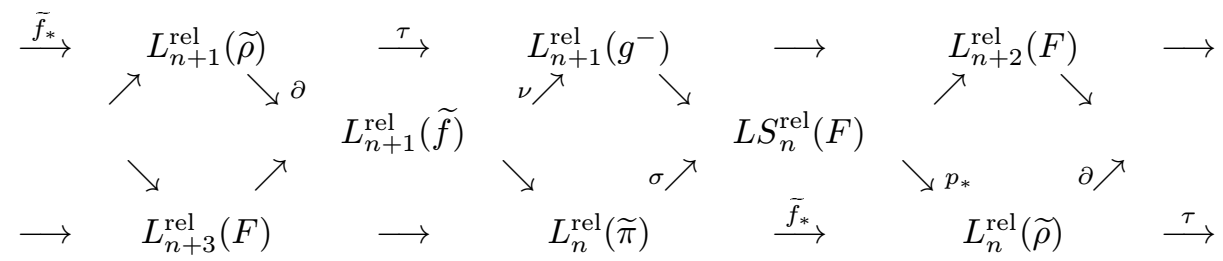

By Lemma 4.15 the map $\widetilde{f}_{*}$ is an epimorphism for all $n$. Hence the map $\partial$ is trivial. Furthermore, $\tau=\nu \partial=0$ and we obtain the short exact sequence

$$
0 \rightarrow L_{n+1}^{\mathrm{rel}}\left(g^{-}\right) \rightarrow L S_{n}^{\mathrm{rel}}(F) \stackrel{p_{\text {*ै }}}{\rightarrow} L_{n}^{\mathrm{rel}}(\widetilde{\rho}) \rightarrow 0 .
$$

Let $\delta: L_{n}^{\text {rel }}(\widetilde{\rho}) \rightarrow L_{n}^{\text {rel }}(\widetilde{\pi})$ be the right inverse of the map $\widetilde{f}_{*}$. It is well defined by Lemma 4.15. Now, $p_{*} \circ(\sigma \circ \delta)=\left(p_{*} \circ \sigma\right) \circ \delta=\widetilde{f}_{*} \circ \delta=\mathrm{Id}$, that is, the map $\sigma \circ \delta$ splits the map $p_{*}$, and the proof of the theorem is complete.

Theorem 4.18. Under the hypotheses of Theorem 4.7 there exist natural isomorphisms

$$
L P_{n}^{\mathrm{rel}}(F)=L_{n+1}^{\mathrm{rel}}\left(g^{-}\right) \oplus L_{n+1}^{\mathrm{rel}}\left(j_{-}^{!}\right) \quad \text { for each } n=0,1,2,3 \bmod 4 .
$$

Proof. Consider the diagram (3.14) for relative $L$-groups:

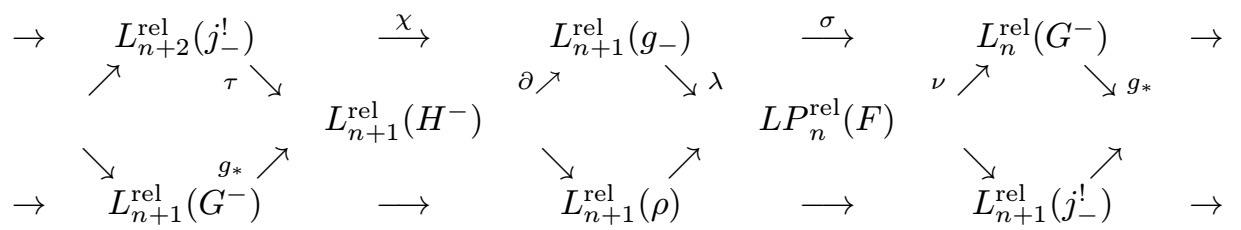

By Lemma 4.15 the map $g_{*}$ is an epimorphism for all $n$. Hence the map $\partial$ is trivial. Furthermore, $\chi=\partial \circ \tau=0$ and we obtain the short exact sequence

$$
0 \rightarrow L_{n+1}^{\mathrm{rel}}\left(g^{-}\right) \stackrel{\lambda}{\rightarrow} L P_{n}^{\mathrm{rel}}(F) \rightarrow L_{n+1}^{\mathrm{rel}}\left(j_{-}^{!}\right) \rightarrow 0 .
$$

As follows from Lemma 4.15, a left inverse $p: L_{n}^{\mathrm{rel}}\left(G^{-}\right) \rightarrow L_{n+1}^{\mathrm{rel}}\left(g_{-}\right)$of the map $\sigma$ is well defined. Hence $(p \circ \nu) \circ \lambda=p \circ(\nu \circ \lambda)=p \circ \sigma=\operatorname{Id}$ and the map $p \circ \nu$ is the left inverse of $\lambda$. This completes the proof.

We consider now the natural map $\Upsilon: F \rightarrow \Psi$ of geometric diagrams of groups

$$
\left(\begin{array}{ccc}
(\pi, w, \theta, b, \varepsilon) & \stackrel{f}{\rightarrow}\left(\rho, w^{\prime}, \theta^{\prime}, b^{\prime}, \varepsilon^{\prime}\right) \\
\downarrow i & \downarrow j \\
(G, w, \theta, b, \varepsilon) \stackrel{g}{\rightarrow}\left(H, w^{\prime}, \theta^{\prime}, b^{\prime}, \varepsilon^{\prime}\right)
\end{array}\right) \stackrel{\Upsilon}{\longrightarrow}\left(\begin{array}{c}
\left(\rho, w^{\prime}, \theta^{\prime}, b^{\prime}, \varepsilon^{\prime}\right) \rightarrow\left(\rho, w^{\prime}, \theta^{\prime}, b^{\prime}, \varepsilon^{\prime}\right) \\
\downarrow i \\
\downarrow j \\
\left(H, w^{\prime}, \theta^{\prime}, b^{\prime}, \varepsilon^{\prime}\right) \rightarrow\left(H, w^{\prime}, \theta^{\prime}, b^{\prime}, \varepsilon^{\prime}\right)
\end{array}\right) .
$$


The map $\Upsilon$ induces the natural maps of the $L S$ - and $L P$-groups. Then by Theorems 4.17 and 4.18 we have the splitting short exact sequences

$$
\begin{aligned}
& 0 \rightarrow L_{n+1}^{\mathrm{rel}}\left(g^{-}\right) \rightarrow L S_{n}^{\mathrm{rel}}(F) \rightarrow L S_{n}^{\mathrm{rel}}(\Psi) \rightarrow 0, \\
& 0 \rightarrow L_{n+1}^{\mathrm{rel}}\left(g^{-}\right) \rightarrow L P_{n}^{\mathrm{rel}}(F) \rightarrow L P_{n}^{\mathrm{rel}}(\Psi) \rightarrow 0 .
\end{aligned}
$$

Recall that

$$
\begin{aligned}
& L S_{*}^{p}(\Psi) \cong L N_{*}^{p}(\rho \rightarrow H)=L_{*}^{p}(\widetilde{\rho}), \quad\left[L P_{*}^{p}(\Psi) \cong L_{*+1}^{p}\left(j_{-}^{!}\right),\right. \\
& L S_{*}^{p}\left(\Psi_{2}\right) \cong L N_{*}^{p}\left((\rho \rightarrow H)_{2}\right)=L_{*}^{p}\left(\widetilde{\rho}_{2}\right), \quad L P_{*}^{p}\left(\Psi_{2}\right) \cong L_{*+1}^{p}\left(\left(j_{-}^{!}\right)_{2}\right) .
\end{aligned}
$$

Similar results hold for the "rel"-groups.

We can now compute all natural maps in the diagram (3.16) for $L^{\text {rel }}$-groups.

Theorem 4.19. The map $\Upsilon$ induces the natural decomposition of the diagram

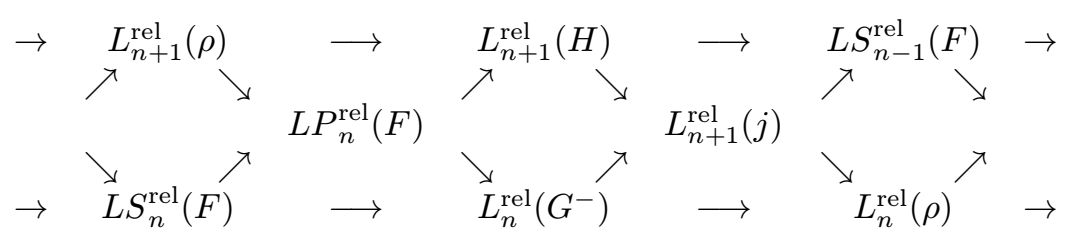

into the direct sum of the two diagrams

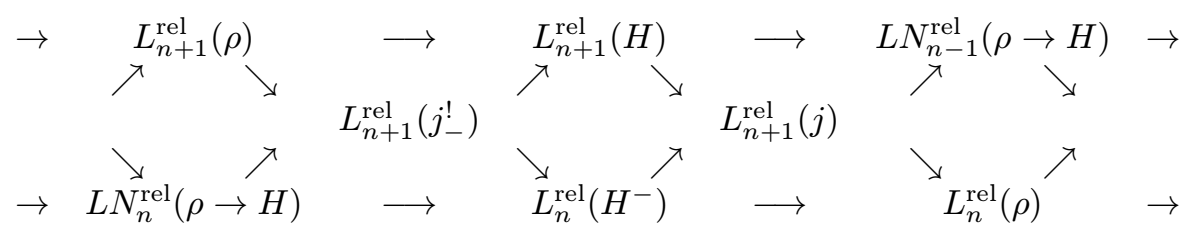

and

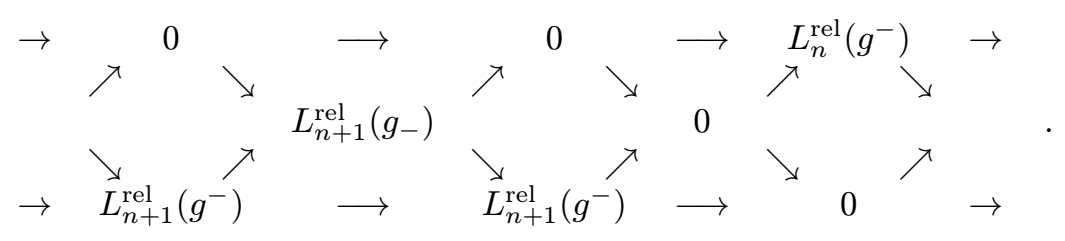

Recall that in the case of "rel"-groups the maps in the diagram (3.16) for the square $\Psi$ were computed in [12].

The exact sequence (4.3) and Theorem 4.7 give one the exact sequences

$$
0 \rightarrow L S_{2 n+1}^{\mathrm{rel}}(F) \stackrel{\partial_{2 n+1}}{\rightarrow} L S_{2 n}^{p}(F) \stackrel{\sigma}{\rightarrow} \underbrace{\|_{\mathbb{Z} / 2} S_{2 n}^{p}\left(F_{2}\right)}_{\|} \stackrel{\psi}{\rightarrow} L S_{2 n}^{\mathrm{rel}}(F) \stackrel{\partial_{2 n}}{\rightarrow} L S_{2 n-1}^{p}(F) \rightarrow 0
$$

where $F$ is the geometric diagram (3.1) and $n=0,1 \bmod 2$. 
Note that by [12] one obtains the exact sequences

$$
0 \rightarrow L_{2 n+1}^{\mathrm{rel}}(\widetilde{\rho}) \stackrel{\partial_{2 n+1}^{\prime}}{\rightarrow} L_{2 n}^{p}(\widetilde{\rho}) \stackrel{\sigma^{\prime}}{\rightarrow} \underbrace{L_{2 n}^{p}\left(\widetilde{\rho}_{2}\right)}_{\mathbb{\mathbb { Z }} / 2} \stackrel{\psi^{\prime}}{\rightarrow} L_{2 n}^{\mathrm{rel}}(\widetilde{\rho}) \stackrel{\partial_{2 n}^{\prime}}{\rightarrow} L_{2 n-1}^{p}(\widetilde{\rho}) \rightarrow 0
$$

for $n=0,1 \bmod 2$, and similar results hold for all other geometric antistructures considered above. Moreover, the map $\psi^{\prime}$ is computed in [12]. We shall apply these results to the computation of the maps $\psi$ in (4.20).

Let $\psi_{\tilde{\rho}}=\psi^{\prime}$; we shall consider similar maps

$$
\begin{gathered}
\psi_{G^{-}}: \mathbb{Z} / 2 \rightarrow L_{2 n}^{\mathrm{rel}}\left(G^{-}\right), \\
\psi_{H^{-}}: \mathbb{Z} / 2 \rightarrow L_{2 n}^{\mathrm{rel}}\left(H^{-}\right), \\
\psi_{\tilde{\pi}}: \mathbb{Z} / 2 \rightarrow L_{2 n}^{\mathrm{rel}}(\widetilde{\pi})
\end{gathered}
$$

for $n=0,1 \bmod 2$.

Theorem 4.22. If at least one of the maps $\psi_{\bar{\rho}}, \psi_{G^{-}}$, and $\psi_{H^{-}}$is a monomorphism, then the map $\psi$ in the exact sequence (4.20) is also a monomorphism.

Proof. The diagram (3.9) is natural. Hence there exist maps of (4.20) into similar exact sequences for the groups $G^{-}, H^{-}$, and $\widetilde{\rho}$. For example, in the case of the group $\widetilde{\rho}$ we obtain the commutative diagram

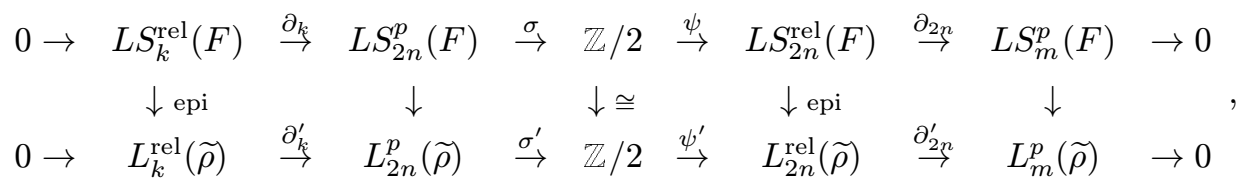

where $k=2 n+1$ and $m=2 n-1$. The vertical maps here are induced by $\Upsilon$. We now obtain the result in the case when $\psi_{\tilde{\rho}}$ is one-to-one. All other cases can be discussed in a similar manner.

Theorem 4.23. If the map $\psi_{\tilde{\pi}}$ is trivial, then $\psi$ in the exact sequence (4.20) is also a trivial map.

Proof. In the case of the group $\widetilde{\pi}$ there exists a natural map of the sequence (4.21) into the exact sequence (4.20). The required result can now be obtained similarly to the proof of the preceding theorem.

Theorem 4.24. Under the hypotheses of Theorem 4.7 there exists an exact sequence

$0 \rightarrow L_{2 n+2}^{\mathrm{rel}}\left(g^{-}\right) \rightarrow L S_{2 n}^{p}(F) \stackrel{\Upsilon_{*}}{\rightarrow} L_{2 n}^{p}(\widetilde{\rho}) \stackrel{\Delta}{\rightarrow} L_{2 n+1}^{\mathrm{rel}}\left(g^{-}\right) \rightarrow L S_{2 n-1}^{p}(F) \stackrel{\Upsilon_{*}}{\rightarrow} L_{2 n-1}^{p}(\widetilde{\rho}) \rightarrow 0$,

where the maps $\Upsilon_{*}$ are induced by the map $\Upsilon, n=0,1 \bmod 2$. The right-hand map $\Upsilon_{*}$ splits, and the map $\Delta$ is either zero or $\operatorname{Im}(\Delta)=\mathbb{Z} / 2$.

Proof. We obtain the required result by extending the vertical rows in the diagram in Theorem 4.22 by exact sequences. 
Remark $4.25 . \operatorname{Im}(\Delta)=\mathbb{Z} / 2$ only in the case when $\psi^{\prime}=0$ and $\psi$ is a monomorphism.

Remark 4.26. The commutative diagram in Theorem 4.22 actually gives one the diagram of exact sequences

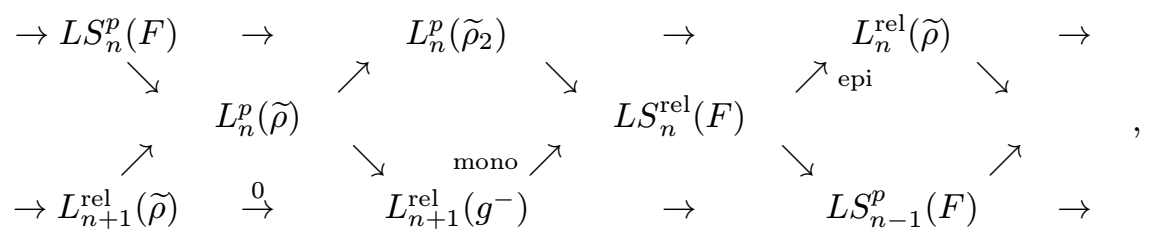

where $L_{n}^{p}\left(\widetilde{\rho}_{2}\right)=\mathbb{Z} / 2,0$ for $n=0,1 \bmod 2$, respectively.

Lemma 4.27. Under the hypotheses of Theorem 4.7 the natural maps

$$
L P_{n}\left(F_{2}\right) \rightarrow L_{n+1}\left(\left(j_{-}^{!}\right)_{2}\right)
$$

from the diagrams (3.14) and (3.15) are isomorphisms for $n=0,1,2,3 \bmod 4$.

Proof. This follows from the diagram (3.15).

Theorem 4.28. Under the hypotheses of Theorem 4.7 there exists a diagram of exact sequences

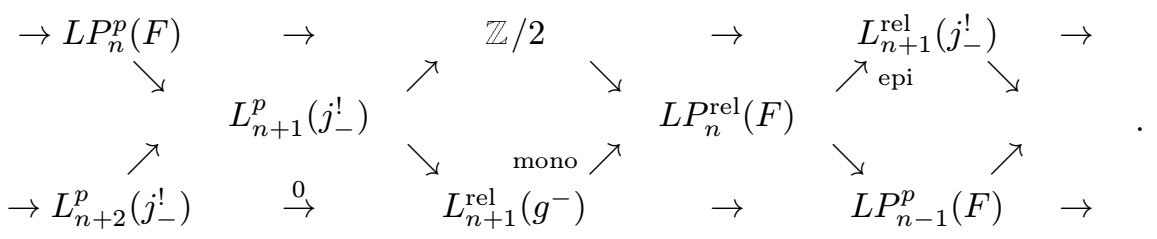

Proof. This follows from Theorems 4.10 and 4.18, Lemma 4.27, and the fact that the diagrams (3.14) and (3.15) are natural.

\section{Bibliography}

[1] C. T. C. Wall, Surgery on compact manifolds, Academic Press, London 1970.

[2] A. A. Ranicki, Exact sequences in the algebraic theory of surgery, Princeton Univ. Press, Princeton, NJ 1981.

[3] W. Browder and G. R. Livesay, "Fixed point free involutions on homotopy spheres", Bull. Amer. Math. Soc. 73 (1967), 242-245.

[4] S. Lopez de Medrano, Involutions on manifolds, Springer-Verlag, Berlin 1971.

[5] S. E. Cappell and J. L. Shaneson, "Pseudo-free actions", Algebraic topology (Aarhus 1978), Lecture Notes in Math., vol. 763, Springer-Verlag, Berlin 1979, pp. 395-447.

[6] I. Hambleton, "Projective surgery obstructions on closed manifolds", Algebraic K-theory, Part II (Oberwolfach 1980) Lecture Notes in Math., vol. 967, Springer-Verlag, Berlin 1982, pp. 101-131.

[7] P. M. Akhmet'ev, "Splitting homotopy equivalences along a one-sided submanifold of codimension 1", Izv. Akad. Nauk SSSR Ser. Mat. 51 (1987), 211-241; English transl. in Math. USSR-Izv. 30 (1988).

[8] A. F. Kharshiladze, "Generalized Browder-Livesay invariant", Izv. Akad. Nauk SSSR Ser. Mat. 51 (1987), 379-401; English transl. in Math. USSR-Izv. 30 (1988).

[9] Yu. V. Muranov, "Obstruction groups to splitting and quadratic extensions of antistructures", Izv. Ross. Akad. Nauk Ser. Mat. 59:6 (1995), 107-132; English transl. in Izv. Math. 59 (1995). 
[10] Yu. V. Muranov and D. Repovš, "Groups of obstructions to surgery and splitting for a manifold pair", Mat. Sb. 188:3 (1997), 127-142; English transl. in Sb. Math. 188 (1997).

[11] A. Ranicki, "The L-theory of twisted quadratic extensions", Canad. J. Math. 39 (1987), $345-364$.

[12] I. Hambleton, L. R. Taylor, and B. Williams, "An introduction to maps between surgery obstruction groups", Algebraic topology (Aarhus 1982), Lecture Notes in Math., vol. 1051, Springer-Verlag, Berlin 1984, pp. 49-127.

[13] I. Hambleton, L. R. Taylor, and B. Williams, "Detection theorems for K-theory and L-theory", J. Pure Appl. Algebra 63 (1990), 247-299.

[14] C. T. C. Wall, "On the classification of Hermitian forms. VI. Group rings", Ann. of Math. (2). 103 (1976), 1-80.

[15] I. Hambleton and I. Madsen, "On the computation of the projective surgery obstruction groups", $K$-theory 7 (1993), 537-574.

[16] C. T. C. Wall, "Formulae for surgery obstructions", Topology 15 (1976), 189-210.

[17] S.P. Novikov, "Algebraic construction and properties of Hermitian analogs of K-theory over rings with involution from the viewpoint of Hamiltonian formalism. Applications to differential topology and theory of characteristic classes, I, II", Izv. Akad. Nauk SSSR Ser. Mat. 34 (1970), 253-288; English transl. in Math. USSR-Izv. 4 (1970).

[18] C. T. C. Wall, "Foundations of algebraic L-theory", Algebraic K-theory, III (Seattle, WA 1972), Lecture Notes in Math., vol. 343, Springer-Verlag, Berlin 1973, pp. 266-300.

[19] C. T. C. Wall, "On the axiomatic foundation of the theory of Hermitian forms", Proc. Cambridge Philos. Soc. 67 (1970), 243-250.

[20] I. Hambleton, A. Ranicki, and L. Taylor, "Round L-theory", J. Pure Appl. Algebra 47 (1987), 131-154.

[21] Yu. V. Muranov, "Relative Wall groups and decorations", Mat. Sb. 185:12 (1994), 79-100; English transl. in Russian Acad. Sci. Sb. Math. 83 (1995).

Vitebsk State Technical University;

McMaster University, Ontario, Canada

E-mail address: mur@vstu.unibel.by; hamblton@mcmail.CIS.McMaster.CA

Received 12/JAN/99

Translated by Yu. MURANOV 